\title{
Effect of individualized psychological intervention on negative emotion and sleep quality of patients after bladder cancer surgery: a randomized controlled trial
}

\author{
Peng Zhang ${ }^{1 \#}$, Yu Zhang ${ }^{2 \#}$, Xiuwu Han ${ }^{1}$, Xin Zhang', Xuhui Zhu', Tao Li ${ }^{1}$ \\ ${ }^{1}$ Department of Urology, Beijing Chao-Yang Hospital, Beijing, China; ${ }^{2}$ Department of Respiratory and Critical Care Medicine, Beijing Chao-Yang \\ Hospital, Capital Medical University, Beijing, China \\ Contributions: (I) Conception and design: X Han; (II) Administrative support: T Li; (III) Provision of study materials or patients: Y Zhang; (IV) \\ Collection and assembly of data: P Zhang; (V) Data analysis and interpretation: P Zhang, X Zhang, X Zhu; (VI) Manuscript writing: All authors; (VII) \\ Final approval of manuscript: All authors. \\ \#These authors contributed equally to this work. \\ Correspondence to: Xiuwu Han. Director of Department of Urology, Beijing Chaoyang Hospital, Capital Medical University (western campus), Beijing \\ 100043, China. Email: xiuwuhan@163.com.
}

Background: Bladder surgery has a negative impact on patients' mental health, sleep quality. Conventional nursing interventions failed to meet the clinical needs. Therefore, this parallel-group RCT was performed to assess the effect of individualized psychological intervention on patients' negative emotions and sleep quality after bladder cancer surgery.

Methods: Patients with bladder cancer admitted to our hospital (from September 2018 to October 2020) and underwent surgical treatment were selected as the research objects. They were randomly allocated to a parallel control group or observation group based on a computer-generated random numbers table. Because our study included behavioral intervention, blinding was not possible for participants and care givers. The control group received conventional nursing care, and the observation group received strengthened individualized psychological intervention under the nursing measures of the control group. Sleep quality, anxiety, depression, nursing compliance, and the occurrence of bladder spasm were compared between the two groups.

Results: Seventy-eight eligible patients were included and randomly divided in two groups ( $\mathrm{n}=39$ for each group). The intervention procedure was completed without losses and exclusions of patients after randomization. Before the intervention, the Pittsburgh Sleep Quality Index Scale and the anxiety and depression scores were comparable between the two groups $(\mathrm{P}>0.05)$. After the intervention, the data of the two groups were significantly improved. The sleep quality, sleep time, sleep efficiency, the time to fall asleep, hypnotic drugs, sleep disorders, daytime dysfunction, anxiety score, and depression score of the observation group were lower than those of the control group, with significant differences $(\mathrm{P}<0.05)$. The total nursing compliance rate of the observation group reached $100.00 \%$, which was higher than the control group. The difference between the groups was significant $(\mathrm{P}<0.05)$. There was no significant difference in the incidence of bladder spasm between the two groups $(\mathrm{P}>0.05)$.

Conclusions: Individualized psychological intervention applied to bladder cancer surgery can effectively improve patients' negative emotions and improve their sleep quality.

Trial registration: Chinese Clinical Trial Registry ChiCTR2100046468.

Keywords: Individualized psychological intervention; bladder cancer; negative emotions; sleep quality

Submitted May 25, 2021. Accepted for publication July 14, 2021.

doi: 10.21037/tau-21-534

View this article at: https://dx.doi.org/10.21037/tau-21-534 


\section{Introduction}

As an important muscular cystic organ in the urinary system, the bladder has a high probability of developing malignant tumors. In recent years, the incidence of bladder cancer has been increasing year by year (1). Clinically, bladder cancer is divided into muscular invasive type and non-muscular invasive type. The treatments are mainly partial cystectomy and total cystectomy. The operation changes the normal way of urination, which causes certain inconvenience to the patient's life. Surgery has a negative impact on patients' mental health, affects their sleep quality and quality of life, and is not conducive to a good recovery from the disease. Coupled with surgical stimulation and invasive operations, it further increases the sensitivity of the bladder, easily causing adverse events, such as bladder spasm and infection (2), thus adversely affecting the physical and mental health of patients. Conventional nursing interventions cannot meet the actual clinical needs Hence, how to use clinical nursing to help patients effectively regulate their own emotions, improve sleep quality, and further improve quality of life and disease prognosis are problems that need to be solved urgently.

This article studied 78 cases of bladder cancer patients underwent surgical treatment in our hospital from September 2018 to October 2020. This study aimed to explore the application of individualized psychological interventions for patients underwent bladder cancer surgery. Investigating the impact on their negative emotions and sleep quality could lay the foundation for clinical practical work. We present the following article in accordance with the CONSORT reporting checklist (available at https:// dx.doi.org/10.21037/tau-21-534).

\section{Methods}

\section{Information}

\section{Inclusion and exclusion criteria}

The inclusion criteria were as follows: those who were diagnosed with bladder cancer by imaging, pathological examination, and clinical manifestations (3); those who were tolerant to surgical treatment; those who were 18 years old and above; those who had obtained informed consent and actively cooperated.

The exclusion criteria were as follows: those with severe basic diseases; those with mental abnormalities or impaired consciousness; those with dysfunction of important organs such as the liver and kidneys; those with malignant diseases of other systems or organs.

\section{Information source}

According to the inclusion and exclusion criteria, this parallel-group RCT study collected patients who underwent bladder cancer surgery in our hospital from September 2018 to October 2020, and the cases were uniformly reviewed by the project expert group. A total of 78 cases were included. According to the random number table method, they were divided into the control group and the observation group ( $n=39$ for each group) with a computer generated 1:1 randomization sequence with varying block size generated by a statistician not participating this project. Project nurses blinded to block size randomized participants using numbered, sealed, and opaque envelopes. Because our study included behavioral intervention, blinding was not possible for participants and care givers. While those assessing the outcomes were blinded to group assignment. Gender, age, medical history, and medication history of each patient were registered. All procedures performed in this study involving human participants were in accordance with the Declaration of Helsinki (as revised in 2013). The study was approved by institutional ethics committee board of Beijing Chao-Yang Hospital (No.2018-Science-302) and informed consent was taken from all the patients.

\section{Study methods}

The control group received routine nursing, and the patients were informed of the relevant precautions after admission. Patients cooperated with the doctor to complete all operations, and their vital signs and surgical incisions were closely monitored. Relevant precautions were relayed to patients and family members. They were also told to regularly clean and disinfect, and keep the ward clean and tidy. Related questions from patients and family members were patiently answered. Furthermore, patients were informed to maintain proper temperature, humidity, and good ventilation, maintain a low-salt and low-fat diet, appropriately increase the intake of protein, vitamins, etc., and avoid cold stimulation and spicy food.

Under the nursing measures of the control group, the observation group had strengthened individualized psychological intervention, specifically:

(I) a nursing team was established consisting of 2 nurses and 1 doctor. Information on the patient's age, personality, condition, family environment, education level, etc., was collected. Their psychological status was assessed and 
targeted nursing plans were formulated, providing an experience exchange platform. Group activities and health knowledge lectures were carried out once a week.

(II) Upon admission, patients and their family members were actively informed of the ward system to help patients familiarize themselves with the hospital environment as soon as possible. Empathy, talking, guidance, and other skills were used to communicate with patients to eliminate their nervousness and strangeness and to quickly establish a good nurse-patient relationship. Furthermore, psychological hints, encouraging language, successful case presentations, etc., were used to help patients build confidence in treatment. The appropriate nursing language was chosen according to the patient's education level. Health education was strengthened through one-toone, one-to-many, etc. Patients and their families were informed about the basic procedures, expected results, precautions, and other knowledge of the operation with the help of pictures, videos, and brochures, thus further correcting their misunderstandings, eliminating their concerns, and improving the degree of patient cooperation. These measures were conducted 10 minutes a day for 3 consecutive days.

(III) Before surgery, patients often experience a series of negative emotions due to fear of surgery accidents, surgery results, or economic burden, which can cause loss of appetite, sleep disturbances, etc. These worries will affect the patient's tolerance to surgery, which is not conducive to a smooth operation. Therefore, at this time, it is necessary to strengthen the popularization of surgical safety knowledge, and help patients understand the safety of surgery, the meaning of benign psychology, and active cooperation of the treatment effect through personal statements, etc. It is also necessary to arouse their attention, pay attention to changes in the patient's language and behavior, evaluate their psychological status, and eliminate or reduce their negative emotions through music therapy and attention shifting, among other methods. These measures were conducted twice a day, each time for 10 minutes and for 3 consecutive days.

(IV) After the operation, we actively communicated with the patients and family members, and comforted patients in reasonable way to avoid adverse consequences if the operation failed to achieve the desired results. If the operation was successfully completed, the patient and family members were informed of the results of the operation as soon as possible, and were again informed of the importance of postoperative care and co-treatment, improving their self-care ability, and guiding the patient to exercise and eat a reasonable diet. For patients with postoperative pain, soothing music was played for them, paying attention that it was not too loud, and patients were guided to read books, watch TV, play chess, and undertake other activities to divert their attention. Pain relievers were given if necessary. These measures were conducted once a day for 5 minutes each time and continued until hospital discharge.

\section{Follow-up}

There were 39 cases in both groups, with persistent observation. Sleep quality, anxiety, and depression were primary endpoint events of this trail and they were assessed at the time of admission and before discharge. The secondary endpoints were defined as nursing compliance and the occurrence of bladder spasm of the patients.

Sleep quality was evaluated by the Pittsburgh Sleep Quality Index Scale, with a total score of 21 points and a low score as the advantage. There were 7 components: sleep quality, sleep time, sleep efficiency, time to fall asleep, hypnotics, sleep disorders, and day dysfunction.

Anxiety and depression were evaluated by the Anxiety Self-Rating Scale and Depression Self-Rating Scale. The highest score was 100 points. The higher the score, the higher the degree of negative emotion. The positive standard for the anxiety scale was $\geq 50$ points, and the positive standard for the depression scale was $\geq 53$ points.

Nursing compliance was divided into 3 levels: high compliance, basic compliance, and non-compliance. High compliance indicated full compliance with the doctor's advice to complete the various nursing operations. Basic compliance indicated being able to cooperate with the completion of nursing operations after persuasion, and non-compliance indicated refusing to cooperate with related nursing operations. Total compliance rate $=$ high compliance + basic compliance.

Bladder spasm was divided into 3 grades according to the severity: mild, moderate, and severe. Mild indicated that there was hematuria leakage from the catheter and no obvious color change of the washing fluid. Moderate represented that the bladder had a feeling of fullness and there was mild spasmodic distention pain and leakage of blood and urine from the urethral catheter. In severe cases, there was an urge to urinate, and severe cramping pain in the lower abdomen. The patient's breath-holding occurred once every few minutes, the blood color of the washing fluid deepened, and the washing fluid refluxed. 


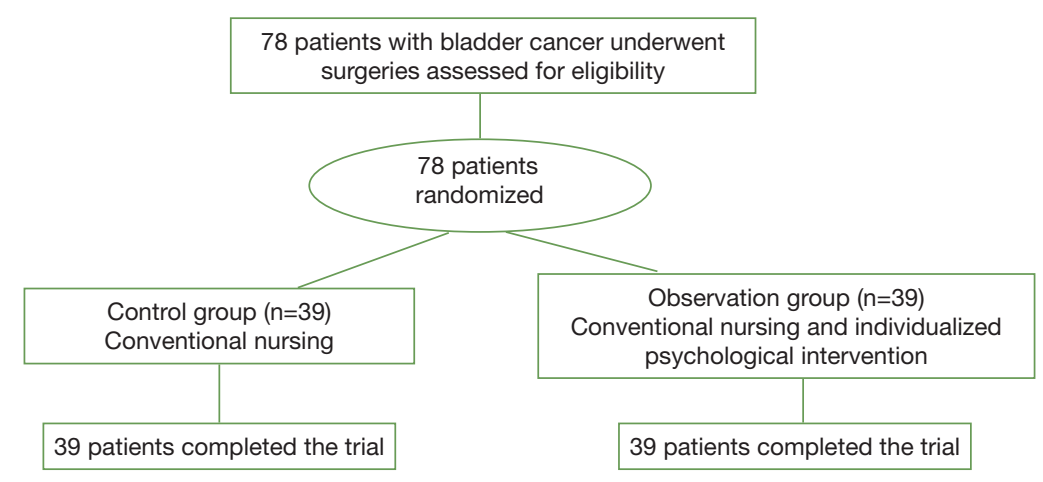

Figure 1 Participant flow chart.

Table 1 Analysis of baseline data in the 2 groups

\begin{tabular}{|c|c|c|c|c|}
\hline Item & Observation group $(n=39)$ & Control group $(n=39)$ & $t / \chi^{2}$ & $\mathrm{P}$ \\
\hline Male & 20 & 24 & 0.83 & 0.36 \\
\hline Female & 19 & 15 & & \\
\hline Age & $38-71(58.91 \pm 4.03)$ & $41-73(60.02 \pm 5.69)$ & 0.99 & 0.32 \\
\hline Non-muscle invasive type & 36 & 34 & 0.56 & 0.46 \\
\hline Muscle invasive bladder cancer & 3 & 5 & & \\
\hline \multicolumn{5}{|l|}{ Degree of education } \\
\hline Junior high school and below & 22 & 19 & 0.47 & 0.79 \\
\hline
\end{tabular}

\section{Statistical analysis}

The sample size ( $\mathrm{n}=78$ patients) was calculated via GPower 3.1 software through power analysis for the primary outcome (patients' negative emotions and sleep quality) to allow for $10 \%$ dropout. Data was analyzed by SPSS 20.0 software. Count data were expressed as percentages (sleep quality score, anxiety score, depression score), and the chisquare test was used. Continuous data were expressed as mean \pm standard deviation (nursing compliance, occurrence of bladder spasm), and the $t$-test was used. $\mathrm{P}<0.05$ indicated that the comparison was statistically significant.

\section{Results}

\section{Baseline information}

From September 2018 to October 2020, a total of 78 eligible patients with bladder cancer were recruited in our hospital and divided into control group and the observation group ( $\mathrm{n}=39$ for each group). No patient losses and exclusions after randomization in the two groups (Figure 1). There was no significant difference in gender, age, and disease type between the two groups $(\mathrm{P}>0.05)$, as shown in Table 1. 
Table 2 The Pittsburgh Sleep Quality Index Scale scores in the 2 groups (n, points)

\begin{tabular}{|c|c|c|c|c|c|c|c|c|}
\hline Group & $\begin{array}{l}\text { Sleep } \\
\text { quality }\end{array}$ & $\begin{array}{l}\text { Sleep } \\
\text { time }\end{array}$ & $\begin{array}{c}\text { Sleep } \\
\text { efficiency }\end{array}$ & $\begin{array}{c}\text { Sleep } \\
\text { latency }\end{array}$ & $\begin{array}{l}\text { Hypnotic } \\
\text { drugs }\end{array}$ & $\begin{array}{c}\text { Sleep } \\
\text { disorders }\end{array}$ & $\begin{array}{c}\text { Daytime } \\
\text { dysfunction }\end{array}$ & Total score \\
\hline \multicolumn{9}{|l|}{ Observation group $(n=39)$} \\
\hline After & $0.87 \pm 0.30$ & $1.60 \pm 0.29$ & $0.95 \pm 0.25$ & $0.79 \pm 0.33$ & $0.68 \pm 0.17$ & $0.87 \pm 0.36$ & $1.59 \pm 0.44$ & $7.34 \pm 1.05$ \\
\hline \multicolumn{9}{|l|}{ Control group $(n=39)$} \\
\hline Before & $1.91 \pm 0.18$ & $2.39 \pm 0.41$ & $1.90 \pm 0.58$ & $1.70 \pm 0.48$ & $1.11 \pm 0.34$ & $1.93 \pm 0.63$ & $2.50 \pm 0.71$ & $13.50 \pm 1.76$ \\
\hline After & $1.50 \pm 0.49$ & $2.08 \pm 0.67$ & $1.27 \pm 0.39$ & $1.25 \pm 0.32$ & $0.92 \pm 0.28$ & $1.34 \pm 0.58$ & $2.19 \pm 0.63$ & $9.22 \pm 1.24$ \\
\hline $\mathrm{P}$ (comparison before intervention) & 0.29 & 0.59 & 0.86 & 0.35 & 0.16 & 0.51 & 0.27 & 0.70 \\
\hline$t$ (comparison after intervention) & 6.85 & 4.11 & 4.31 & 6.25 & 4.58 & 4.30 & 4.88 & 7.23 \\
\hline $\mathrm{P}$ (comparison after intervention) & 0.00 & 0.00 & 0.00 & 0.00 & 0.00 & 0.00 & 0.00 & 0.00 \\
\hline
\end{tabular}

Table 3 Anxiety and depression scores in the 2 groups

\begin{tabular}{|c|c|c|c|c|c|c|c|c|}
\hline Group & \multicolumn{4}{|c|}{ Anxiety score (points) } & \multicolumn{4}{|c|}{ Depression score (points) } \\
\hline Observation group $(n=39)$ & $52.38 \pm 4.09$ & $22.07 \pm 3.56$ & 34.91 & 0.00 & $50.98 \pm 3.74$ & $21.96 \pm 4.88$ & 29.48 & 0.00 \\
\hline Control group $(n=39)$ & $54.05 \pm 6.26$ & $39.71 \pm 6.06$ & 10.28 & 0.00 & $51.62 \pm 3.38$ & $35.69 \pm 2.24$ & 24.53 & 0.00 \\
\hline$t$ & 1.39 & 15.67 & - & - & 0.79 & 15.97 & - & - \\
\hline
\end{tabular}

\section{Comparison of sleep quality between the two groups}

Sleep quality was observed in the two groups, at the time of admission and before discharge. The Pittsburgh Sleep Quality Index Scale scores before the intervention of the two groups were comparable $(\mathrm{P}>0.05)$. After the intervention, the scores of the two groups were significantly reduced. The sleep quality, sleep time, sleep efficiency, sleep time, hypnotic drugs, sleep disturbance, daytime dysfunction scores, and total score were $0.87 \pm 0.30$, $1.60 \pm 0.29,0.95 \pm 0.25,0.79 \pm 0.33,0.68 \pm 0.17,0.87 \pm 0.36$, $1.59 \pm 0.44$, and $7.34 \pm 1.05$ points, respectively, which were lower than the control group. The difference between the groups was significant $(\mathrm{P}<0.05)$, as shown in Table 2 .

\section{Comparison of anxiety and depression between the two groups}

The anxiety and depression scores of the two groups before the intervention were comparable $(\mathrm{P}>0.05)$. After the intervention, the anxiety and depression scores of the observation group were $22.07 \pm 3.56$ and $21.96 \pm 4.88$ points, respectively, which were lower than those of the control group. The difference was significant $(\mathrm{P}<0.05)$, as shown in Table 3. 
Table 4 The total compliance rate of nursing care in the 2 groups

\begin{tabular}{lcccc}
\hline Group & High compliance & Basic compliance & No compliance & Total compliance rate $(\%)$ \\
\hline Observation group $(\mathrm{n}=39)$ & 25 & 14 & 0 & 100.00 \\
Control group $(\mathrm{n}=39)$ & 20 & 13 & 6 & 84.62 \\
$\chi^{2}$ & - & - & - & 6.50 \\
$\mathrm{P}$ & - & - & - & 0.01 \\
\hline
\end{tabular}

Table 5 Incidence of bladder spasm in the 2 groups

\begin{tabular}{lcccc}
\hline Group & Mild & Moderate & Severe & Total $(n, \%)$ \\
\hline Observation group $(\mathrm{n}=39)$ & 5 & 3 & 0 & $8(20.51)$ \\
Control group $(\mathrm{n}=39)$ & 6 & 5 & 2 & $13(33.33)$ \\
$\chi^{2}$ & - & - & - & 1.63 \\
$\mathrm{P}$ & - & - & - & 0.20 \\
\hline
\end{tabular}

\section{Comparison of nursing compliance between the two groups}

The total nursing compliance rate of the observation group reached $100.00 \%$, which was higher than the control group. The difference between the groups was significant $(\mathrm{P}<0.05)$, as shown in Table 4.

\section{Comparison of the occurrence of bladder spasm between the two groups}

The incidence of bladder spasm in the observation group was $20.51 \%$, which was lower than that in the control group, but the difference between the two groups was not significant $(\mathrm{P}>0.05)$, as shown in Table 5 .

\section{Discussion}

Bladder cancer is a common malignant disease of the urinary system, which has a serious impact on the physical and mental health of patients $(4,5)$. Patients often show great psychological burden and stress after being diagnosed with this disease. They not only suffer from the stress from cancer development and recurrence, but also current surgery caused adverse psychological reactions. Different degrees of negative emotions, such as anxiety and depression, can be seen in the treatment and rehabilitation stage. The appearance of negative emotions will not only cause changes in patients' physical function, and affect their normal sleep and quality of life (6), but it also affects their treatment and nursing compliance, which is not conducive to disease treatment. The patient may even have thoughts and behaviors to commit suicide. Therefore, it is of great significance to actively implement effective nursing intervention while providing necessary surgical treatment to patients with bladder cancer. These measures are conducive to disease recovery and can help improve patients' negative emotions, sleep, and quality of life (7-9).

The treatment of cancer lies not only in the disease itself and prolonging survival time, but also in improving quality of life $(10,11)$. Long-term continuous negative emotions will affect the endocrine system, central nervous system, and other functions, causing many undesirable consequences $(12,13)$. In this article, the control group adopted the traditional clinical nursing model, and the observation group had strengthened individualized psychological intervention on the basis of the routine nursing model. The emphasis of traditional nursing intervention is usually limited to the disease or treatment itself and predispose to ignore individual need and physical feeling of the patient, in spite that recent nursing also progressively emphasizes psychological intervention. The traditional nursing activities are often passive and cannot meet the actual clinical needs. While, the individualized psychological intervention is a novel nursing mode centered on patients, which is personalized, creative and holistic. Compared with the conventional nursing mode, individualized nursing intervention concentrates on active service and communication, which providing multi-dimensional and indepth nursing services according to patients' personalized needs.

Furthermore, the individualized nursing practice make comprehensive and specific schedules throughout all the treatment stages based on features of the patients. A dedicated nursing team effectively communicated with patients using empathy, talking, and other skills, and comprehensively evaluated the patient's condition, family background, personality, and education level. Targeted 
nursing programs were developed to help patients correcting misunderstandings, eliminating worries, and enhancing treatment confidence through psychological hints and popularization of health knowledge. Then, the degree of cooperation in treatment and nursing was improved, allowing patients to feel a warm and harmonious atmosphere. The patients were encouraged and supported by music therapy, knowledge popularization, and speaking out according to different situations before and after the operation. An experience exchange platform was also provided to guide patients and to encourage each other, strengthen communication, maintain an optimistic attitude, and relax physically and mentally, which is of positive significance for improving the condition of the disease and speeding up the recovery.

Besides, for patients with muscular-invasive or nonmuscular bladder cancer, different psychological intervention skills should be used since they have different disease severity and stages, surgical procedure and prognosis. Patients with muscular-invasive bladder cancer are prone to have higher clinical stages, higher risk of distant metastasis and are mainly treated with radical cystectomy. Therefore, they are exposed to great stress not only from the disease itself and the possible poor prognosis, but also total cystectomy caused discomfort and urination change caused inconvenience, which may result in more severe psychological problems. For these patients, psychological interventions should focus on eliminating extreme thoughts and behavior of the patients, helping them to build confidence to fight against the disease, further to relieve inferiority, despair and other adverse psychological reactions. For patients with nonmuscular invasive bladder cancer, the psychological reactions are relatively moderate. These patients mainly worry about the surgical procedure and recurrence of bladder cancer as well as the pain caused by bladder chemical perfusion after the surgery. In this context, health lecture, individualized emotion support, and experience communication should be provided to reduce the patient's psychological stress and to increase their ability to resist the pressure.

Pittsburgh Sleep Quality Index Scale and anxiety and depression scores were comparable between the two groups before the intervention. After the intervention, the two groups had significant improvements. The sleep quality, sleep time, sleep efficiency, the time to fall asleep, hypnotic drugs, sleep disorders, daytime dysfunction, anxiety score, and depression score of the observation group were significantly lower than those of the control group. In the current study, 6 patients exhibited poor compliance and all these events occurred in the control group with conventional interventions. While the patients in the observation group all showed high or basic compliance. These clinical data indicated the advantage of personalized nursing strategy in improving patients' nursing compliance. For the patients with poor compliance, we should analyze its cause and try to inform the importance of routine physiology and psychological care to the patients and their family members to improve their long-term prognosis. In terms of the incidence of bladder spasm, the observation group had a lower incidence than the control group, but no statistical difference was shown between the two groups. This may be related to the sample size, which should be expanded for further in-depth study. This study showed the effectiveness and feasibility of individualized psychological intervention. Adhering to the people-oriented principle and emphasizing human dignity are the fundamental requirements of modern nursing concepts $(14,15)$. These findings indicate that effective psychological intervention can significantly alleviate patients' negative emotions.

In summary, individualized psychological intervention has significant effects on patients after bladder cancer surgery. This measure can effectively alleviate patients' negative emotions, improve their sleep quality, and is of benefit for the prognosis of the disease. It is therefore worthy of further promotion and application. It also should be noted that the specific psychological interventions would increase the burden of nursing staffs to some extent. Therefore, in the future clinical nursing practice, the actual need for specific psychological interventions in patients should be assessed prior to the procedure to improve the efficiency of this intervention and to ensure a reasonable application. Besides, although the psychological intervention procedures are mainly performed by medical and nursing staffs, some assisting work should be conducted to support the nursing practice, like preparing short videos or handbooks and building communicating platform to share related medical knowledge, treatment strategies, nutrition support and rehabilitation skills. Moreover, nursing staff should be familiar with the intervention procedure, have a deep understanding on the concept of personalized care and are proficiency in using the specific psychological interventions skills.

\section{Acknowledgments}

Funning: This work was mainly supported by the randomized controlled clinical study of hyperthermia 
combined with intravesical infusion of gemcitabine in the treatment of high risk superficial bladder cancer (PX2019011).

\section{Footnote}

Reporting Checklist: The authors have completed the CONSORT reporting checklist. Available at https://dx.doi. org/10.21037/tau-21-534

Trial Protocol: Available at https://dx.doi.org/10.21037/tau21-534

Data Sharing Statement: Available at https://dx.doi. org/10.21037/tau-21-534

Conflicts of Interest: All authors have completed the ICMJE uniform disclosure form (available at https://dx.doi. org/10.21037/tau-21-534). The authors have no conflicts of interest to declare.

Ethical Statement: The authors are accountable for all aspects of the work in ensuring that questions related to the accuracy or integrity of any part of the work are appropriately investigated and resolved. All procedures performed in this study involving human participants were in accordance with the Declaration of Helsinki (as revised in 2013). The study was approved by institutional ethics committee board of Beijing Chao-Yang Hospital (No.2018Science-302) and informed consent was taken from all the patients.

Open Access Statement: This is an Open Access article distributed in accordance with the Creative Commons Attribution-NonCommercial-NoDerivs 4.0 International License (CC BY-NC-ND 4.0), which permits the noncommercial replication and distribution of the article with the strict proviso that no changes or edits are made and the original work is properly cited (including links to both the formal publication through the relevant DOI and the license). See: https://creativecommons.org/licenses/by-nc-nd/4.0/.

\section{References}

1. Bolat D, Çelik S, Aydın ME, et al. Assessment of the quality of life and sexual functions of patients followedup for non-muscle invasive bladder cancer: preliminary results of the prospective-descriptive study. Turk J Urol 2018;44:393-8.

2. Goltz HH, Major JE, Goffney J, et al. Collaboration Between Oncology Social Workers and Nurses: A PatientCentered Interdisciplinary Model of Bladder Cancer Care. Semin Oncol Nurs 2021;37:151114.

3. Bladder cancer: diagnosis and management of bladder cancer: (C) NICE (2015) Bladder cancer: diagnosis and management of bladder cancer. BJU Int 2017;120:755-65.

4. Hamad J, McCloskey H, Milowsky MI, et al. Bladder preservation in muscle-invasive bladder cancer: a comprehensive review. Int Braz J Urol 2020;46:169-84.

5. Black AJ, Black PC. Variant histology in bladder cancer: diagnostic and clinical implications. Transl Cancer Res 2020;9:6565-75.

6. Jung A, Nielsen ME, Crandell JL, et al. Quality of Life in Non-Muscle-Invasive Bladder Cancer Survivors: A Systematic Review. Cancer Nurs 2019;42:E21-E33.

7. Lavdaniti M, Zyga S. Quality of Life in Elderly Bladder Cancer Patients Following a Cystectomy. Adv Exp Med Biol 2017;989:297-300.

8. Masiero M, Busacchio D, Guiddi P, et al. Quality of life and psycho-emotional wellbeing in bladder cancer patients and their caregivers: a comparative analysis between urostomy versus ileal orthotopic neobladder. Ecancermedicalscience 2021;15:1163.

9. Zang Y, Li X, Cheng Y, et al. An overview of patients with urothelial bladder cancer over the past two decades: a Surveillance, Epidemiology, and End Results (SEER) study. Ann Transl Med 2020;8:1587.

10. Mogavero MP, DelRosso LM, Fanfulla F, et al. Sleep disorders and cancer: State of the art and future perspectives. Sleep Med Rev 2021;56:101409.

11. Choi H, Park JY, Bae JH, et al. Health-related quality of life after radical cystectomy. Transl Androl Urol 2020;9:2997-3006.

12. Rammant E, Van Wilder L, Van Hemelrijck M, et al. Health-related quality of life overview after different curative treatment options in muscle-invasive bladder cancer: an umbrella review. Qual Life Res 2020;29:2887-910.

13. Singh S, Eguchi M, Min SJ, et al. Outcomes of Patients With Cancer Discharged to a Skilled Nursing Facility After Acute Care Hospitalization. J Natl Compr Canc Netw 2020;18:856-65.

14. Yu EY, Nekeman D, Billingham LJ, et al. Health-related 
quality of life around the time of diagnosis in patients with bladder cancer. BJU Int 2019;124:984-91. Erratum in: BJU Int 2020;126:646.

15. Zhao B, Wang K, Jiang B. Application of clinical

Cite this article as: Zhang P, Zhang Y, Han X, Zhang X, Zhu X, $\mathrm{Li}$ T. Effect of individualized psychological intervention on negative emotion and sleep quality of patients after bladder cancer surgery: a randomized controlled trial. Transl Androl Urol 2021;10(7):3021-3029. doi: 10.21037/tau-21-534 nursing pathway in bladder cancer patients undergoing ureteroperitoneostomy. Panminerva Med 2020. doi: 10.23736/S0031-0808.20.04010-0. [Epub ahead of print]. 